\title{
Open versus laparoscopic appendicectomy in acute appendicitis: results of a district general hospital
}

\author{
D Zosimas ${ }^{1}$, P M Lykoudis ${ }^{2}$, A Pilavas ${ }^{1}$, J Burke, ${ }^{1}$ P Leung ${ }^{1}$, G Strano ${ }^{1}$, V Shatkar ${ }^{1}$ \\ ${ }^{1}$ Department of General Surgery, Queen's Hospital, Romford, Essex, UK \\ ${ }^{2}$ University College London Hospital, London, UK
}

Corresponding author: Dimitrios Zosimas (dizos@yahoo.com)

\begin{abstract}
Background: Despite multiple studies comparing the two methods, the real advantages of laparoscopic appendicectomy (LA) compared to open appendicectomy (OA) are still unclear. Purpose of the current study was to compare the results between the two techniques in a district general hospital.

Methods: The electronic records of all patients who underwent OA or LA in a one year period were reviewed retrospectively. The comparative data points assessed included age, gender, overall complications, length of stay and Clavien-Dindo Classification of Surgical Complications, including the rates of the main types of complications.

Results: 300 patients were included in the study. 166 patients underwent OA and 134 patients LA. Postoperative complications were documented in 26 patients $(8.7 \%)$. LA was employed predominantly in female patients $(p=0.004)$ and in older patients $(\mathrm{p}=0.0015)$ and was associated with significantly more negative appendicectomies than $\mathrm{OA}(\mathrm{p}=0.002)$. No statistically significant difference was noted with regards to the length of hospital stay $(\mathrm{p}=0.577)$, overall postoperative morbidity $(p=0.543)$ and grading of complications $(p=0.460)$. Finally, following comparison of the incidence of specific types of complications, only wound infections were significantly different, in favour of LA.

Conclusions: LA is safe and effective, however, besides the lower incidence of wound sepsis, demonstrates no clear advantage over OA. The selection between OA and LA should thus be tailored to the clinical scenario and the surgeon's preference.
\end{abstract}

S Afr J Surg 2018;56(2)

http://dx.doi.org/10.17159/2078-5151/2018/v56n2a2392

\section{Introduction}

Appendicitis is the most common acute surgical diagnosis worldwide. Open appendicectomy (OA) has been the gold standard treatment for more than 100 years, because of its proven safety and efficacy. With the introduction of minimally invasive endoscopic surgery, laparoscopic appendicectomy (LA) has become increasingly popular and is claimed to be safe and superior to OA in terms of hospital stay, postoperative pain, wound complications, diagnostic abdominal exploration, return to normal activities and cosmetic result, including demonstrated advantages in obese patients with regards to hospital stay and overall complications. ${ }^{1-3}$ These findings have been challenged by other authors who observed no significant difference in the outcomes between the two procedures and moreover noted higher costs and longer operative times with LA. ${ }^{4,5}$ Although safe, LA is not free of complications or specific adverse events such as intra-abdominal abscesses (IAA). ${ }^{6}$ Due to prolonged overall surgery duration, high incidence of postoperative IAA and rate of conversion to OA, there is doubt as to the suitability of laparoscopy in complicated appendicitis, which requires advanced laparoscopic skills. ${ }^{7,8}$

Despite wide experience of many centres and multiple prospective and retrospective studies, the advantages of LA compared to OA are still either unclear ${ }^{9,10}$ or of limited clinical relevance ${ }^{8}$ and have prevented LA from completely replacing $\mathrm{OA}$ and being advocated as the procedure of choice for appendicectomy. The aim of the current study was to compare the results of open and laparoscopic appendicectomies with regards to safety at a district general hospital.

\section{Materials and methods}

The electronic records of all patients who underwent open or laparoscopic appendicectomies in a one year period in a single institution were reviewed retrospectively. Laparoscopic procedures converted to open were included in the open group. The cases in which complicated ovarian cysts or other pathologies were identified during surgery or in which an appendicectomy was performed during other primary 
procedure were excluded. The demographics of the patients were documented. The parameters studied were age, gender, morbidity, length of stay in the hospital (LOS) and surgical histology. Morbidity included any postoperative incidents deviating from the standard postoperative recovery, pain of such severity as to require review at the Accident and Emergency Department or readmission to the hospital or further postoperative imaging and finally readmissions for any other reason related to the initial operation, including cases in which a reoperation was needed. A stratification of the complications was also attempted following the ClavienDindo Classification of Surgical Complications (C-D). ${ }^{11}$ Surgical histology was defined as negative when no evidence of appendicitis or other pathology was identified in the specimen and as positive when the specimen demonstrated evidence of appendicitis or other pathologic findings that could explain the patient's symptoms. All the cases of acute appendicitis were stratified according to The American Association of Surgery for Trauma (AAST) grading scale for emergency general surgery conditions. ${ }^{12}$ Median length of follow up was 19 months (range 13-24). Bivariate correlations between scale and binomial variables were assessed using Mann-Whitney U test. Correlations of categorical variables in 4-fold tables were assessed using Fisher's exact test (2-tailed) and in $>4$-fold table using chi-square test (2-tailed). A p-value of less than 0.05 was considered statistically significant. Statistical processing of data was conducted using SPSS v20 software (IBM Corporation, Chicago, IL, USA). The study was conducted in full compliance with local Ethical Regulations and Anonymization standards. Approval from local ethical committee was not required as the study involved only retrospective analysis of clinical data associated with diagnostic and therapeutic techniques performed without any deviation from institute's local guidelines. Due to the retrospective nature of the study, it was not necessary to obtain informed consent from patients who had already been consented for the operative procedure.

The authors of this study have no conflict of interest to declare and no funding sources have supported this research.

\section{Results}

A total of 311 patients underwent an appendicectomy in a one year period in a single centre. 11 patients were excluded from the study: 7 patients had appendicectomy during other primary procedure ( 3 abdominal hysterectomies, 2 sigmoid colectomies, 1 small bowel resection, 1 prostatectomy), 3 patients had appendicectomy during an ovarian cystectomy and for 1 patient the appendix was not included in the specimen. Of the 300 patients included in the study, $145(48.3 \%)$ patients were male and $155(51.7 \%)$ patients were female. There were 166 patients who underwent OA and 134 patients who had LA. In 10 cases a conversion from laparoscopic to open procedure was performed. The median age at time of surgery was 27 years. Negative final histology was found in 69 patients. The median length of stay was 3 days.
Postoperative complications were documented in 26 patients (Table 1), which are elaborated on in Table 2, including one patient requiring re-operation for persistent abdominal pain (stump appendicitis ). Based on the C-D classification, 9 patients had grade I complications, 13 patients grade II and 4 patients grade III. There were no patients with grade IV or grade $\mathrm{V}$ complications (Table 1). The laparoscopic approach was employed predominantly in female patients $(p=0.004)$ and in older patients $(p=0.0015)$. It was also found to be significantly associated with more negative appendicectomies than OA $(p=0.002)$. No statistically significant difference was observed regarding the length of stay $(p=0.577)$ and overall postoperative morbidity $(\mathrm{p}=0.543$ ). This was confirmed by comparing the severity of complications by means of the C-D classification of complications $(p=0.460)$, including a further comparison by classifying the C-D grades into two groups $($ I versus $>$ I) $(\mathrm{p}=0.399)$ (Table 3$)$. In a comparison of complications such as wound infection, IAA, postoperative pain and ileus, wound infections were the only significant difference, in favour of LA (Table 4). Per AAST grading scale, grade 1 prevailed (54.7\%) followed by grade $3(23.5 \%)$, grade $2(16.9 \%)$ and grade $4(3.3 \%)$, while grade 5 accounted for $1.6 \%$ of the cases. The grading system demonstrated statistically significant bivariate correlations with the length of stay $(p=0.003)$, with categories 4 and 5 being associated with longer hospitalisation, but not with the overall complications $(p=0.121)$. Finally, although the AAST grading scale did correlate statistically significantly with the incidence of wound infections $(p=0.001)$, it did not demonstrate any statistically significant correlation with the type of operation (OA or LA) $(\mathrm{p}=0.353)$, thus no further examination for the role of AAST grading scale as a confounding factor was considered necessary. Therefore, the result in favour of LA in comparison to OA with regards to the incidence of wound infections can be further validated.

\begin{tabular}{lcc}
\multicolumn{3}{l}{ Table 1: Distribution of demographics and outcomes } \\
\hline Demographics - Outcomes & N (\%) & $\begin{array}{c}\text { Median } \\
\text { (range) }\end{array}$ \\
Male patients & $145(48.3)$ & \\
Female patients & $155(51.7)$ & \\
Age (time of surgery) & & \\
Open appendicectomy & $166(5-93)$ \\
Laparoscopic appendicectomy & $134(44.7)$ & \\
Length of hospital stay(days) & & \\
Clavien-Dindo Grade of & & \\
Complications & \\
I & $9(34.6)$ \\
II & $13(50.0)$ \\
III & $4(15.4)$ \\
Postoperative complications & $26(8.7)$ \\
Conversion to open surgery & $10(6.9)$ \\
Negative Final Histology & $69(23.0)$
\end{tabular}


Table 2: Breakdown of complications

\begin{tabular}{ll}
\hline Complications (type) & Number of patients \\
Pain & 11 \\
Wound infection & 12 \\
Intra-abdominal collection/abscess & 6 (1 drained) \\
Ileus & 2 \\
Lung consolidations & 1 \\
Pleural effusion & 1 (drained) \\
Acute Kidney Injury & 2 \\
Abdominal wall haematoma & 1 \\
Reoperation & 1
\end{tabular}

Table 3: Comparison of demographics and outcomes across surgical approaches

\begin{tabular}{|c|c|c|c|}
\hline $\begin{array}{l}\text { Demographics - } \\
\text { Outcomes }\end{array}$ & Laparoscopic & Open & P-value \\
\hline \multicolumn{4}{|l|}{ Gender * } \\
\hline Male & $52(38.8)$ & $93(56.0)$ & \multirow{2}{*}{0.004} \\
\hline Female & $82(61.2)$ & $73(44.0)$ & \\
\hline Age (time of surgery) ${ }^{\dagger}$ & $28(14-67)$ & $25(6-93)$ & 0.015 \\
\hline Length of stay(days) ${ }^{\dagger}$ & $3(1-14)$ & $3(1-53)$ & 0.577 \\
\hline \multicolumn{4}{|l|}{$\begin{array}{l}\text { Clavien - Dindo grades } \\
\text { of complications }\end{array}$} \\
\hline I & $2(20.0)$ & $7(43.8)$ & \multirow[b]{3}{*}{0.460} \\
\hline II & $6(60.0)$ & $7(43.8)$ & \\
\hline III & $2(20.0)$ & $2(12.5)$ & \\
\hline \multicolumn{4}{|c|}{$\begin{array}{l}\text { Clavien-Dindo groups of } \\
\text { grades of complications" }\end{array}$} \\
\hline I & $2(20.0)$ & $7(43.8)$ & \multirow{2}{*}{0.399} \\
\hline$>\mathrm{I}$ & $8(80.0)$ & $9(56.2)$ & \\
\hline \multicolumn{4}{|c|}{ Postoperative morbidity ${ }^{*}$} \\
\hline Yes & $10(7.5)$ & $16(9.6)$ & \multirow{2}{*}{0.543} \\
\hline No & $124(92.5)$ & $150(90.4)$ & \\
\hline \multicolumn{4}{|l|}{ Final histology* } \\
\hline Positive & $92(68.7)$ & $139(83.7)$ & \multirow[b]{2}{*}{0.002} \\
\hline Negative & $42(31.3)$ & $27(16.3)$ & \\
\hline $\begin{array}{l}{ }^{*} \mathrm{~N}(\%) \\
{ }^{\dagger} \text { median (range) }\end{array}$ & & & \\
\hline
\end{tabular}

Table 4: Comparison of complications across surgical approaches

\begin{tabular}{llll}
\hline Complications * & $\begin{array}{l}\text { Open } \\
(\mathbf{n = 1 6 6 )}\end{array}$ & $\begin{array}{l}\text { Laparoscopic } \\
(\mathbf{n = 1 3 4 )}\end{array}$ & P-value \\
$\begin{array}{l}\text { Wound infection } \\
12(7.2 \%)\end{array}$ & 0 & 0.001 \\
$\begin{array}{l}\text { Intra-abdominal } \\
\text { collection/abscess }\end{array}$ & $4(2.4 \%)$ & $2(1.5 \%)$ & 0.695 \\
Pain & $7(4.2 \%)$ & $4(3 \%)$ & 0.760 \\
Ileus & $2(1.2 \%)$ & 0 & 0.504 \\
${ }^{*} \mathrm{~N}(\%)$ & & &
\end{tabular}

\section{Discussion}

Despite numerous previous studies the superiority of LA remains unclear, particularly for complicated appendicitis, as many of these show similar rates of overall complications. There is a lower incidence of wound infections with LA and the LOS appears to be shorter, however the incidence of IAA and the cost of the procedure is higher. Despite these small differences, the superiority of LA may not translate into clinical relevance. ${ }^{1,13-15}$

In our study, OA correlated better with the final histology of appendicitis in keeping with current literature., ${ }^{915-17}$ This is most likely due to laparoscopy being preferred as a primarily diagnostic procedure in equivocal cases. LA was more likely to be performed in female patients in the current study, in keeping with other studies. , $8,13,16,18,19$ Patients who underwent OA were younger compared to LA. This could be explained by the inclusion of paediatric population in the present study. Results in children look similar to adults according to different studies. ${ }^{14}$ No significant difference between the two approaches has been revealed regarding overall complications and this has been further supported by the comparison of the C-D grading of complications. The findings for overall complications reported in the literature are variable with some studies, like ours, showing no difference in overall postoperative complications, ${ }^{1,7,8,16,20}$ and others showing significantly lower complications for LA. ${ }^{4,5,18,21}$ With regards to the incidence of wound infections, although variations in definition exist, most of the recent studies concur that the incidence is significantly reduced with LA. ${ }^{1,2,4,7,14,17-20,22}$ This is in keeping with the results of the present study. The sheathed ports used in laparoscopic surgery and in lesser extend the smaller size of incisions along with the placement of the appendix in a bag, reduces the risk for wound infection. A recent Cochrane systematic review, supported by other studies, reported that the incidence of IAA in OA was lower compared to LA. ${ }^{2,7,8,13,14,22}$ The lack of laparoscopic experience in treating complicated appendicitis and the inadequacy of abdominal lavage, along possibly with non-inversion of the stump, exposure of the infectious source to the whole intra-abdominal space and dissemination due to pneumoperitoneum are mechanisms that could explain this higher incidence in LA. ${ }^{2,7}$ However this was not of concern in the present study as no statistical difference was demonstrated between the two techniques, in agreement with other reports. ${ }^{4,5,17,19,20}$ It is likely that meticulous irrigation of the peritoneal cavity laparoscopically, with change of position of the patient and use of copious amount of normal saline, along with the improvement of laparoscopic technique can explain this improvement of outcomes. ${ }^{22}$ In the present study, authors did not find any significant difference in postoperative pain between the two procedures. Great variability exists in literature, ${ }^{2,415,20}$ partly due to heterogeneity in definition and assessment of pain and variety of analgesics. ${ }^{14}$ With regards to postoperative ileus it has been reported that it is more common in OA compared to LA, ${ }^{2,4,22}$ likely because of laparoscopic surgeon's ability to minimise manipulation of the cecum and 
ileum..$^{22}$ However this was not the case in the present study as no statistical difference was proved.

The question of whether LA decreases the length of hospitalisation has been a matter of great debate over the past years. Although LOS has been reported significantly shorter in LA in recent studies, $, 1,4,5,7,14,16,18-20,22$ there was no difference in the LOS in the current study between the two approaches on the side of other reports. ${ }^{6,8,17,21}$ This can be explained by the small sample size of the study, by the fact that wound infections are mainly managed in the community and the outpatient setting therefore they would not affect the LOS and by the not significant difference in the incidence of ileus, postoperative pain and IAA between the two groups, all factors that would affect the LOS. Nevertheless, comparison of different studies with regards to LOS remains difficult due to inclusion of diagnostic laparoscopies in some studies on LOS, differences related to different social standards, insurance systems and patient's and surgeon's expectations ${ }^{8,15}$ and variability in the definition of LOS.

In the current study, bias in selection of technique by the surgeons has not been considered, neither have the experience of the surgeons, the availability of LA after hours and other factors which could affect the outcomes. The rate of laparoscopic appendicectomies converted to open was $6.9 \%$ in this study, which is comparable to the literature $(6.4-8 \%)^{7,8,15}$ The conversion was based on subjective intraoperative criteria and inability to proceed laparoscopically in relation to severe complicated appendicitis (8 cases) or extensive adhesions ( 2 cases), in the context that our unit has been regularly performing laparoscopic appendicectomies for about 10 years. The inclusion of converted procedures in the OA group, in line with other studies ${ }^{9}$ and the decision whether or not to remove a normal appendix may also have affected the results. On the other hand, great variability of outcomes exists in literature, explained by heterogeneity of studies, differing exclusion criteria ${ }^{1,5,7,13,21}$ and the fact that in some studies complications after discharge and ambulatory care were not taken into account. ${ }^{18}$

Although in later series LA yields better results, there is probably little to offer to improve outcomes as OA is already of minor invasiveness and short LOS, considering that in the current study open appendicectomies were performed predominantly via a local incision (McBurney or Lanz incisions with relative modifications) and only in 5 cases of complicated appendicitis midline laparotomies were performed. LA appears to offer further advantages regarding cosmesis, reduction of the incidence of adhesions, ability of irrigation of the abdomen and performance of a diagnostic laparoscopy, particularly in obesity, ${ }^{5,10,15}$ but on the other hand presents disadvantages of longer operative times, higher costs, a higher incidence of IAA and lack of availability after hours. ${ }^{14,17}$ In the present study, the incidence of specific complications and LOS were similar between the two procedures, except for the reduced incidence of wound infections in favour of LA. We therefore conclude that LA cannot be deemed clearly superior to OA.

\section{Conclusions}

Within the limitations of a retrospective study, LA in a district general hospital is shown to be safe and effective but not clearly advantageous over OA, except for its lower incidence of wound infections. The issue regarding IAA highlighted in literature even though not present in this study needs to be delineated and further research should be focused on this topic as well as on determining the true advantages of LA, ideally with larger prospective randomised controlled trials. If laparoscopic equipment and relative expertise are available then it can be used, particularly in young female obese patients. ${ }^{14}$ Until this debate has been settled in the literature, the choice of appendicectomy modality should be guided by institutional policy, the clinical presentation and the skill of the surgeon.

\section{REFERENCES}

1. Liu Z, Zhang P, Ma Y, et al. Laparoscopy or not: a metaanalysis of the surgical effects of laparoscopic versus open appendicectomy. Surg Laparosc Endosc Percutan Tech. 2010;20(6):362-70.[10.1097/SLE.0b013e3182006f40]

2. Bennett J, Boddy A, Rhodes M. Choice of approach for appendicectomy: a meta-analysis of open versus laparoscopic appendicectomy. Surg Laparose Endosc Percutan Tech. 2007;17(4):245-55.[10.1097/SLE.0b013e318058a117]

3. Varela JE, Hinojosa MW, Nguyen NT. Laparoscopy should be the approach of choice for acute appendicitis in the morbidly obese. Am J Surg. 2008;196(2):218-22.[10.1016/j. amjsurg.2007.08.067]

4. Biondi A, Di Stefano C, Ferrara F, Bellia A, Vacante M, Piazza L. Laparoscopic versus open appendectomy: a retrospective cohort study assessing outcomes and cost-effectiveness. World J Emerg Surg. 2016;11(1):44.[10.1186/s13017-016-0102-5]

5. Minutolo V, Licciardello A, Di Stefano B, Arena M, Arena G, Antonacci V. Outcomes and cost analysis of laparoscopic versus open appendectomy for treatment of acute appendicitis: 4-years experience in a district hospital. BMC Surg. 2014;14:14. [10.1186/1471-2482-14-14]

6. Clarke T, Katkhouda N, Mason RJ, et al. Laparoscopic versus open appendectomy for the obese patient: a subset analysis from a prospective, randomized, double-blind study. Surg Endosc. 2011;25(4):1276-80.[10.1007/s00464-010-1359-5]

7. Lim SG, Ahn EJ, Kim SY, et al. A Clinical Comparison of Laparoscopic versus Open Appendectomy for Complicated Appendicitis. J Korean Soc Coloproctol. 2011;27(6):293-7. [10.3393/jksc.2011.27.6.293]

8. Swank HA, Eshuis EJ, van Berge Henegouwen MI, Bemelman WA. Short- and long-term results of open versus laparoscopic appendectomy. World J Surg. 2011;35(6):1221-6; discussion 7-8.[10.1007/s00268-011-1088-5]

9. Ekeh AP, Wozniak CJ, Monson B, Crawford J, McCarthy MC. Laparoscopy in the contemporary management of acute appendicitis. Am J Surg. 2007;193(3):310-3; discussion 3-4. [10.1016/j.amjsurg.2006.09.025]

10. Khalil J, Muqim R, Rafique M, Khan M. Laparoscopic versus open appendectomy: a comparison of primary outcome measures. Saudi J Gastroenterol. 2011;17(4):236-40. [10.4103/1319-3767.82574]

11. Dindo D, Demartines N, Clavien PA. Classification of surgical complications: a new proposal with evaluation in a cohort of 6336 patients and results of a survey. Ann Surg. 
2004;240(2):205-13.

12. Tominaga GT, Staudenmayer KL, Shafi S, et al.The American Association for the Surgery of Trauma grading scale for 16 emergency general surgery conditions: Disease-specific criteria characterizing anatomic severity grading. J Trauma Acute Care Surg. 2016;81(3):593-602.[10.1097/TA.0000000000001127]

13. Ingraham AM, Cohen ME, Bilimoria KY, Pritts TA, Ko CY, Esposito TJ. Comparison of outcomes after laparoscopic versus open appendectomy for acute appendicitis at 222 ACS NSQIP hospitals. Surgery. 2010;148(4):625-35; discussion 35-7. [10.1016/j.surg.2010.07.025]

14. Sauerland S, Jaschinski T, Neugebauer EA. Laparoscopic versus open surgery for suspected appendicitis. Cochrane Database Syst Rev. 2010(10):CD001546.[10.1002/14651858.CD001546. pub3]

15. Katkhouda N, Mason RJ, Towfigh S, Gevorgyan A, Essani R. Laparoscopic versus open appendectomy: a prospective randomized double-blind study. Ann Surg. 2005;242(3):439-48; discussion 48-50.

16. Tan WJ, Pek W, Kabir T, Chan WH, Wong WK, Ong HS. Clinical outcome and cost comparison between laparoscopic and open appendicectomy. Ann Acad Med. Singapore 2014;43(9):464-8.

17. Thomson JE, Kruger D, Jann-Kruger C, et al. Laparoscopic versus open surgery for complicated appendicitis: a randomized controlled trial to prove safety. Surg Endosc. 2015;29(7):202732.[10.1007/s00464-014-3906-y]

18. Masoomi H, Mills S, Dolich MO, et al. Comparison of outcomes of laparoscopic versus open appendectomy in adults: data from the Nationwide Inpatient Sample (NIS), 2006-2008. J Gastrointest Surg. 2011;15(12):2226-31.[10.1007/s11605-0111613-8]

19. Yaghoubian A, Kaji AH, Lee SL. Laparoscopic versus open appendectomy: outcomes analysis. Am Surg. 2012;78(10):10836.

20. Ohtani H, Tamamori Y, Arimoto Y, Nishiguchi Y, Maeda $\mathrm{K}$, Hirakawa K. Meta-analysis of the results of randomized controlled trials that compared laparoscopic and open surgery for acute appendicitis. J Gastrointest Surg. 2012;16(10):192939.[10.1007/s11605-012-1972-9]

21. Kouhia ST, Heiskanen JT, Huttunen R, Ahtola HI, Kiviniemi VV, Hakala T. Long-term follow-up of a randomized clinical trial of open versus laparoscopic appendicectomy. Br J Surg. 2010;97(9):1395-400.[10.1002/bjs.7114]

22. Wei HB, Huang JL, Zheng ZH, et al. Laparoscopic versus open appendectomy: a prospective randomized comparison. Surg Endosc. 2010;24(2):266-9.[10.1007/s00464-009-0563-7] 